\title{
Unusual Presentation of Cutaneous Leishmaniasis: Lower Lip Ulcer
}

\author{
Soheila Nasiri ${ }^{1}$, Nikoo Mozafari ${ }^{1,}$, , Fahimeh Abdollahimajd ${ }^{1}$ \\ ${ }^{1}$ Skin Research Center, Shohada-e-Tajrish Hospital, Shahid Beheshti University of Medical Sciences, Tehran, IR Iran \\ ${ }^{*}$ Corresponding author: Nikoo Mozafari, Skin Research Center, Shohada-e-Tajrish Hospital, Tehran, IR Iran. Tel.: +98-2122741509, +98-9122270181, Fax: +98-2122744393, E-mail: Nikoo_
} MD@yahoo.com.

Received: February 07, 2012; Revised: February 21, 2012; Accepted: March 06, 2012

Cutaneous leishmaniasis is a common tropical dermatose with diverse clinical presentations. At times, it may appear in unusual shape or unusual site that causes diagnostic challenge. We reported a case of unusual presentation of cutaneous leishmaniasis which appeared as an ulcer on the lower lip of a ten-year-old boy. The diagnosis was confirmed by identifying leishmania parasites at ulcer smear. The clinical features and treatment options of leishmaniasis will be briefly reviewed.

Keywords: Leishmaniasis; Oral Ulcer; Therapeutics

\section{Introduction}

Cutaneous leishmaniasis is a protozoan-induced disease, caused by numerous leishmania porotozoa species, which are responsible for its clinical diversity (1). Most lesions are typical for leishmaniasis and present as papules, plaques or ulcers located at uncovered areas, particularly at the face and limbs. The common clinical types present no diagnostic difficulties. At times, unusual presentations may cause diagnostic challenges. These include erysipeloid, annular, paronychyal, palmoplantar, sporotrichoid, zosteriform and lip leishmaniasis (2). Lip leishmaniasis, as cutaneous type of leishmaniasis not as part of mucocutaneous type, has been infrequently mentioned in the medical literature. In 1997 only 17 cases of lip leishmaniasis of 2861 patients (0.59\%) were reported from southwest of Iran (3). Although cutaneous leishmaniasis occurs widely throughout Africa, Asia, South America, the Middle East and Mediterranean region, the number of leishmaniasis cases are increasing in the United State and Western Europe due to infection in returning military personnel and international travelers (1). It is therefore important, when a physician encounters an unusual lesion on the lip suspected of leishmaniasis to be able to confirm the diagnosis and manage it properly.

\section{Case Presentation}

A ten-year-old boy presented to the dermatology service with complaint of painless wound on his lower lip. The lesion started 5 months prior as a small nodule in the cen- ter of the lip which slowly extended over a few weeks and then spontaneously ulcerated. It was covered by dried crust. Treatment with antibiotics had not caused any improvement. The patient reported no prior trauma. Examination of the patient showed a $2 \times 3$ centimeter indurated necrotic ulcer on the lower lip, extending to the vermilion. There was purulent discharge from the lesion. No palpable lymph nodes were noted. No cutaneous lesion elsewhere was observed. Systemic examination revealed no abnormality (Figure 1).

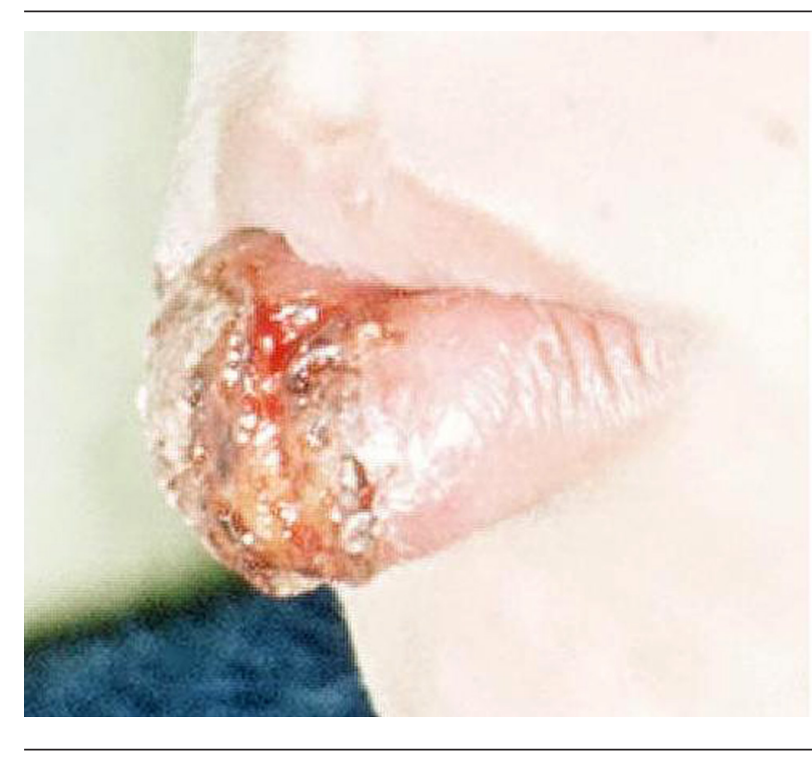

Figure 1. Clinical Presentation of the Lesion on the Lower Lip

Implication for health policy/practice/research/medical education:

Cutaneous leishmaniasis with various clinical presentations may appear in unusual shape or unusual site and causes diagnostic challenge. Therefore, it is recommended to consider cutaneous leishmaniasis in differential diagnosis of any dermatosis which is resistant to conventional treatment, especially in endemic regions.

Copyright @ 2012, Shahid Beheshti University of Medical Sciencces;... This is an Open Access article distributed underthe terms of the Creative Commons Attribution License (http://creativecommons.org/licenses/by/3.0), which permits unrestricted use, distribution, and reproduction in any medium, provided the original work is properly cited. 
With clinical suspicion to cutaneous leishmaniasis, smear was extracted from the margin of ulcerated areaalthough other differential diagnoses like cutaneous tuberculosis, basal cell carcinoma, squamous cell carcinoma, foreign body reaction and fungal infection were also available. In skin smear, leishman bodies were demonstrated (Figure 2).

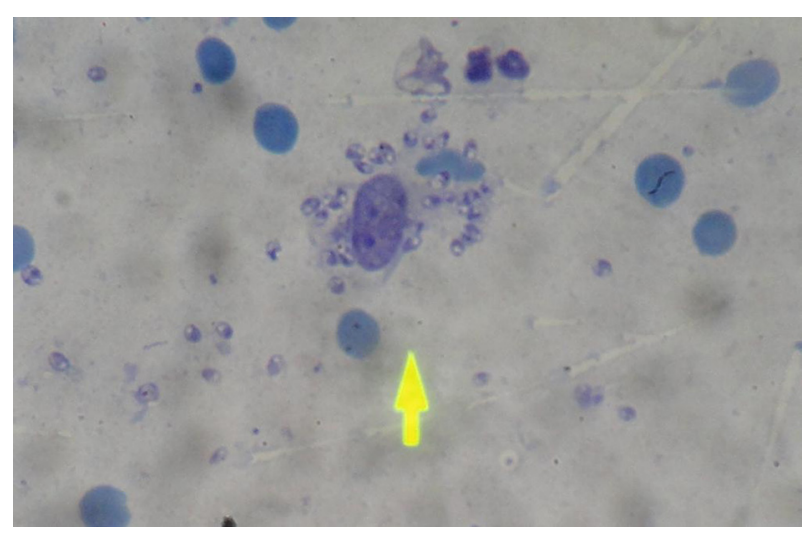

Figure 2. Ulcer Smear Shows Numerous Intra and Extracellular Leishmania Amastigotes (Gimsa Stain $\times 1000$ )

Intramuscular injection of Glucantim $(10 \mathrm{mg} / \mathrm{kg})$ pentavalent antimonial was started for 10 days.

\section{Conclusions}

Leishmaniasis is a protozoan-induced disease that is transmitted through the bite of an infected female sand fly. Notably, more than 20 leishmaniasis porotozoa species are responsible for diseases in humans (1). The disease affects people in areas of southern Europe, South America, Africa and Asia. There has been a marked increase in cases in non-endemic regions as the consequence of increased travel both for tourism and for military personnel returning from Iraq and Afghanistan, where the incidence is high (1). Three clinical forms have been described for leishmaniasis: Cutaneous Leishmaniasis (CL), Mucocutaneous Leishmaniasis (MCL) and Visceral Leishmaniasis (VL). Visceral leishmaniasis is caused by leishmania donovani spp. The earliest lesion is the cutaneous nodule at the site of initial sand fly inoculation, then after parasite attacks the reticuloendothelial system. Clinical symptoms include intermittent fever, hepatosplenomegaly, agranulocytosis, anemia and thrombocytopenia. Susceptibility to secondary infection may produce pulmonary and gastrointestinal infection, leading to death in untreated individuals (4). Mucosal leishmaniasis, characterized by nasooropharangeal mucosal involvement is caused by leishmania Braziliensis spp. It is metastatic complication of cutaneous leishmaniasis. Mucosal disease usually becomes evident several years after original cutane- ous lesions are healed; cutaneous and mucosal lesions can coexist decades apart from one another. It leads to painful destructive ulcer which destroys nasal septum, mouth floor and tonsilar areas; resulting in marked disfigurement (Espundia) (5). Cutaneous leishmaniasis, characterized by one or multiple small erythematous papules, may appear immediately after sand fly bite, but usually 2 to 4 weeks later. Papule slowly enlarges to $2 \mathrm{~cm}$ over a period of several weeks and assumes a dusky violaceous hue. Eventually, the lesion becomes crusted in center with a shallow ulcer and raised indurated border (volcano sign). Small satellite papules at periphery of the lesion and occasionally subcutaneous nodules along the course of proximal lymphatic may develop. Ulcer persists for 3 to 6 months or longer. Then the lesion heals slowly with a slightly depressed scar. Cutaneous leishmaniasis is caused by L. tropica, L. Major and L. aethupica in old world (Asia, Africa, the Middle East,). In new world (Latin America) L. Mexician and L. Brasilian are involved.

Cutaneous leishmaniasis in Iran is predominantly caused by Leishmania Tropica. Leishmaniatropica, in contrast to L. Brazilian rarely, involves the mucous membranes. Few cases of mucosal involvement of L. Tropica due to local spread from a skin lesion near the mouth or the nose have been reported (6). The lip is one of the atypical sites for cutaneous leishmaniasis. Reports from Saudi Arabia (16.7\%) (7) and Turkey (16.2\%) (8) indicate cutaneous leishmaniasis localized to the lip are frequent there; however it is rarely reported in Iran. In 1997, Yaghoobi et al. published a group of 17 patients with cutaneous leishmaniasis localized to the lip from 2,861 patients with $\mathrm{CL}(.59 \%)$ (3). This diversity can be explained in different ways, depending on the lip size of inhabitants; larger lips increase chance of being bitten. Identification of leishmania parasites in dermal macrophages by skin biopsy or dermal scraping can confirm the diagnosis. However, in chronic lesions, parasites may be scarce (9). Therefore, failure to visualize amastigotes on histopathology does not exclude the diagnosis of cutaneous leishmaniasis (1). In this context, the delayed skin reaction test (Montenegro skin test), culture and PCR can be amongst significant diagnostic measurements. These techniques have been proved effective up to $90 \%, 75 \%$, and more than $90 \%$ for patients with cutaneous and mucocutaneous leishmaniasis, respectively (1). When available, PCR appear to be the most sensitive diagnostic test (9). Treatment depends on type and severity of the infection. Infections, according to L. Braziliensis,progress to mucocutaneous disease and therefore require systemic treatment, whereas uncomplicated, localized cutaneous leishmaniasis usually heals spontaneously (9). However, treatment is advocated to not only speed up disease resolution abut also thescar minimization (1).

Pentavalent antimonials $(20 \mathrm{mg} / \mathrm{Kg} /$ day for 14 to 28 
days) remains the standard therapy of cutaneous and mucocutaneous leishmaniasis (9), but the exact dose and length of therapy should be determined for each country. In Iran most cases will respond to $10 \mathrm{mg} / \mathrm{kg}$ for 10 days. Additional therapies include intralesional antimonials, heat therapy, cryotherapy, pentamidine, itraconazole, amphotripcine $\mathrm{B}$, ketoconazole, allopurinol and attractively photodynamic therapy (10) whose efficacy rate varies significantly due to poor clinical trials. In conclusion, a case of cutaneous leishmaniasis with unusual presentation was described. The diagnostic features as well as treatment options for cutaneous leishmaniasis were discussed. This atypical clinical presentation of leishmaniasis should be considered in differential diagnosis of recalcitrant lip lesions by physicians practiced both in endemic and non-endemic areas, since cutaneous leishmaniasis is rising in these regions due to increasing rate of traveling - as tourists or military personnel - to endemic regions.

\section{Acknowledgements}

The authors declared no acknowledgment.

\section{Authors' Contribution}

Data gathering and drafting of the manuscript were performed by Dr Mozafari and Dr Abdollahi-majd. Critical revision of the manuscript was made by Dr Nasiri.

\section{Financial Disclosure}

Authors declare that there is no financial interest.

\section{Funding/Support}

There was no financial support.

\section{References}

1. Ameen M. Cutaneous leishmaniasis: advances in disease pathogenesis, diagnostics and therapeutics. Clin Exp Dermatol. 2010;35(7):699-705.

2. Bari AU, Rahman SB. Many faces of cutaneous leishmaniasis. Italian J Dermatol Venereol Leprol. 2008;74(1):23-7.

3. Yaghoobi R, Hoghooghi-Rad N. Lip leishmaniasis in the Southwest of Iran during the last decade. Iranian J Dermatol.1997:19-23.

4. James WD, Berger T, Elston D. Andrew's Diseases of the Skin: Clinical Dermatology.11 ed.: Elsevier Health Sciences; 2011.

5. Wolff K, Johnson RA. Systemic Parasitic Infections. Fitzpatrick's Color Atlas and Synopsis of Clinical Dermatology. 6 ed.: Mc Graw Hill; 2009.

6. Schewach-Millet M, Kahana M, Ronnen M, Yuzuk S. Mucosal involvement of cutaneous leishmaniasis. Int $J$ Dermatol. 1986;25(2):113-4.

7. El-Hoshy K. Lip leishmaniasis. Journal of the American Academy of Dermatology. 1993;28(4):661-662.

8. Gurel MS, Ulukanligil M, Ozbilge H. Cutaneous leishmaniasis in Sanliurfa: epidemiologic and clinical features of the last four years (1997-2000). Int J Dermatol. 2002;41(1):32-7.

9. Lupi O. Protozoa and Worms. In: Bolognia JL, Jorizzo JL, Rapini RP, editors. Dermatology. 2 ed: Mosby Elsevier; 2008. p.1267.

10. van der Snoek EM, Robinson DJ, van Hellemond JJ, Neumann HA A review of photodynamic therapy in cutaneous leishmaniasis. $J$ Eur Acad Dermatol Venereol. 2008;22(8):918-22. 